IMPLEMENTATION OF VARIABLE METRIC METHODS

FOR CONSTRAINED OPTIMIZATION BASED ON AN

AUGMENTED LAGRANGIAN FUNCTIONAL

N. H. Engersbach

DFVLR Institut für

Dynamik der Flugsysteme

8031 Oberpfaffenhofen, F.R.G.

\author{
W. A. Gruver* \\ Institut für Regelungstechnik \\ Technische Hochschule Darmstadt \\ 6100 Darmstadt, F.R.G.
}

\title{
INTRODUCTION
}

Penalization and gradient projection are two of the simplest and most useful concepts from nonlinear programming. Both provide a means for extending unconstrained gradient descent techniques to accommodate equality and inequality constraints. It is known that penalty function methods may be used to solve a wide class of problems, even those involving nonconvex constraints. In practice, however, the Hessian matrix of the objective functional becomes $i 11$-conditioned, causing convergence difficulties [1]. On the other hand, gradient projection is a concept that involves linear approximation of the constraints and, therefore, is not inherently suited for nonlinear constraints. In the gradient projection algorithm of Rosen [2], which was originally designed for linear constraints forming a bounded convex region, nonlinear constraints were to be accommodated by a restoration step. This procedure is known to be unsatisfactory if a minimum on the tangent plane approximation lies far from the constraint. The difficulty can be avoided by a simple modification of the gradient projection concept that penalizes violations of the constraints along the search direction $[3,4]$.

Recently, Hestenes and Powell suggested that equality constrained minimization problems be reformulated as the unconstrained minimization of an augmented Lagrangian functional involving the sum of terms that are linear and quadratic in the constraint while using independent updates for the Lagrange multiplier $[5,6]$. Some extensions of the concept to inequality constrained optimization have also been obtained, including conditions under which the solution of the dual problem agrees with that of the primal $[7,8]$. An important computational advantage of the augmented Lagrangian concept is that a constrained minimum, if it exists, can be obtained by a finite value of the penalty constant. Computational experience using the technique, al though mostiy for equality constrained problems, has been encouraging $[9,10,11,12,13,14]$.

This paper presents an extension of the modified gradient projection algorithm $[15,16]$, based on an augmented Lagrangian with quadratic penalization. Numerical results from several problems involving static and dynamic, nonlinear equality and inequality constraints are given.

\section{PROBLEM STATEMENT AND NOTATION}

We shall treat the nonlinear programming problem of determining an element $x$ in $R^{n}$ that minimizes a nonlinear functional $f(x)$ subject to nonlinear equality constraints $g(x)=0$ in RP. Inequality constraints will be discussed below under Numerical Results. It will be assumed that $f$ and $g$ are second continuously differentiable and that $a$ relative minimum $\hat{x}$ exists. The gradient $f_{x}$ is an n-tuple, the Jacobian a pxn matrix whose rows are gradients of the elements of $\mathrm{g}$, and $\mathrm{g}_{\mathbf{x}}^{*}$ denotes the transposed matrix. The standard inner product and derived norm on $\mathrm{R}^{n}$ are

$$
<u, v>_{n}=\sum_{i=1}^{n} u_{i} v_{i}, \quad\|u\|_{n}=\left\langle u, u>_{n}^{1 / 2} .\right.
$$

\footnotetext{
*W. A. Gruver is currently with the Department of Chemical Engineering \& Graduate Program in Operations Research at North Carolina State University, Raleigh, North Caroina 27607 USA.
} 


\section{PROJECTION-RESTORATION WITH AN AUGMENTED LAGRANGIAN FUNCTIONAL}

Let $p<n$ and the $p$ gradients of the elements of $g$ be 1 inearly independent. Define the augmented Lagrangian functional

$$
F(x, \lambda, K)=f(x)+\left\langle g, \lambda>_{p}+0.5<g, k g>_{p}\right.
$$

where $\lambda$ is the Lagrange multiplier (a p-tuple), and $k$ is a given pxp diagonal matrix of positive constants. A necessary condition that $F(x, \lambda, K)$ have a minimum for fixed $K$ is that the following equations are satisfied: the constraint equation,

$$
g(\hat{x})=0
$$

and the minimization condition

$$
f_{x}(x)+g_{x}^{*}(\hat{x}) \hat{\lambda}+g_{x}^{*}(\hat{x}) \operatorname{Kg}(\hat{x})=0
$$

An effective approach to the numerical solution of the constrained minimization problem is a two step procedure based on satisfying (2) followed by adjusting $x$ by gradient descent to approximate a solution of $(3)$. The procedure is repeated until a stopping condition is met.

Let the first correction $\delta x$, the restoration increment, to an initial guess $x$ be chosen to satisfy (2) to first order,

$$
g(x)+g_{x}(x) \delta x=0 .
$$

From (4), $\delta x$ may be formally expressed as

$$
\delta x=-g_{x}^{+} g
$$

where, for brevity, the dependence on $x$ has been dropped, and $g_{x}^{+}$is a pseudo inverse of $g_{x}$. Since $p<n$, there exist an infinite number of solutions to (4) of the form $\delta y+\delta z$ where $\delta y$ is any solution of (4) and $\delta z$ is an element in the nullspace of $g_{X}$. Uniqueness can be assured in (5) by use of the minimum norm pseudo inverse

$$
g_{X}^{+}=g_{X}^{*}\left(g_{X} g_{X}^{*}\right)^{-1}
$$

which selects the smallest value of $\delta x$, in the norm on $R^{n}$, lying on the intersection of the tangent planes to the constraints. Linear independence of the gradients of the elements of $g$ ensures that $g_{X} g_{x}^{*}$ is invertible. Using (5), an improved value of $x$ is obtained from

$$
\begin{aligned}
& \bar{x}=x-\hat{\alpha} g_{x}^{*}\left(g_{x} g_{x}^{*}\right)^{-1} g \\
& \hat{\alpha}=\operatorname{argmin}\left\{\left\|g\left(x-\alpha g_{x}^{+} g\right)\right\|_{p} \mid \alpha \text { in } R^{+}\right\} .
\end{aligned}
$$

The second correction $\delta \bar{x}$, the projection increment, is chosen so that $F(\bar{x}, \lambda, k)$ is decreased in the direction of its negative gradient

$$
\delta \bar{x}=-F_{x}(\bar{x}, \lambda, K) \text {. }
$$

The multiplier $\lambda$ needed to compute (8) may be formally expressed, from (3), in terms of a pseudo inverse as

$$
\lambda=-\left(g_{x}^{*}\right)^{+} f_{x}-k g \text {. }
$$

For $x \neq \hat{x}$, there is no value of $\lambda$ in $R^{P}$ for which (3) is satisfied. However, an approximation is obtained by use of the pseudo inverse

$$
\left(g_{X}^{*}\right)^{\frac{*}{*}}=\left(g_{X} g_{X}^{*}\right)^{-1} g_{X}
$$

which selects the least squares value of $\lambda$ in (9). Using (8), (9) and (10), $\bar{x}$ is updated according to the descent iteration

$$
\begin{aligned}
& \overline{\bar{X}}=\bar{x}-\hat{\beta} P f_{X} \\
& \hat{\beta}=\operatorname{argmin}\left\{F\left(\bar{x}-\beta P f_{X}, \lambda, K\right) \mid \beta \text { in } R^{+}\right\}
\end{aligned}
$$

where

$$
P=I-g_{X}^{*}\left(g_{x} g_{x}^{*}\right)^{-1} g_{X}
$$

is an nxn matrix representing the operator that projects the gradient into the manifold formed by the intersection of the constraint tangent planes. 


\section{VARIABLE METRIC IMPLEMENTATION}

For any symmetric, positive definite matrix $G$ define the weighted inner product and derived norm,

$$
<u, v>_{G}=\left\langle u, G v>_{n},\|u\|_{G}=<u, G u>_{n}^{1 / 2}\right.
$$

for $u$ and $v$ in $R^{n}$. Let $H$, the variable metric matrix, be an nxn symmetric, positive definite approximation to the inverse Hessian $F_{x X}^{-1}$, which is assumed to exist. The variable metric matrix generates the minimum norm pseudo inverse

$$
g_{X}^{+}=H g_{x}^{*}\left(g_{X} H g_{X}^{*}\right)^{-1}
$$

which minimizes $\|\delta x\|_{H-1}$, and the least squares pseudo inverse

$$
\left(g_{X}^{*}\right)^{+}=\left(g_{X} H g_{X}^{*}\right)^{-1} g_{X} H,
$$

which minimizes $\left\|f_{x}+g_{x}^{*}+g_{x}^{*} K g\right\|_{H}^{2}$.

Using (13) and (14) the restoration and projection increments are,

$$
\begin{aligned}
\delta x & =-H g_{x}^{*}\left(g_{X} H g_{X}^{*}\right)^{-1} g \\
\delta \bar{x} & =-H\left(f_{X}+g_{X^{\lambda}}^{*}\right) \\
& =-H P f_{X}
\end{aligned}
$$

where

$$
P=I-g_{x}^{*}\left(g_{x} H g_{x}^{*}\right)^{-1} g_{x} H
$$

is an nxn matrix that represents a non-orthogonal projection operator. The variable metric matrix $\mathrm{H}$ is updated after each successful projection update according to established formulas [17]. The use of variable metric descent with gradient projection is due to Goldfarb [18] and Kelley and Speyer [4].

For fixed $K$ suppose that a minimum of $F(x, \dot{\lambda}, K)$ has been obtained. Let the resulting variable metric matrix be $\tilde{H}$. This matrix is now refined according to prescribed changes in the penalty constants $\Delta K=\bar{K}-K$ and the corresponding, al though unknown, changes $\Delta \lambda=\lambda-\lambda$ in the Lagrange multiplier. The update is based on choosing $\bar{\lambda}$ to minimize the modified residual

$$
\left.\| f_{X}+g_{X}^{*}\right)+g_{X}^{*} K g\left\|_{\tilde{H}}^{2}+\right\| \bar{\lambda} \|_{(\Delta K)^{-1}}^{2}
$$

where the second term in (18) is an energy constraint which prevents large changes in the multiplier. If $\Delta K$ is nonsingular, the minimum value of $\lambda$ can be expressed in

closed form as

$$
\bar{\lambda}=-\left(g_{X} H g_{X}^{*}+\Delta K^{-1}\right)^{-1} g_{X} H\left(f_{X}+g_{X}^{*} K g\right) \text {. }
$$

This corresponds to an initial projection increment

$$
\begin{aligned}
& \delta \bar{x}^{(0)}=-H^{(0)}\left(f_{X}+g_{X}^{\star} K g\right) \\
& H^{(0)} \doteq \tilde{H} Q \\
& Q=I-g_{X}^{\star}\left(g_{X} \tilde{H} g_{X}^{*}+\Delta K^{-1}\right)^{-1} g_{X} \tilde{H} .
\end{aligned}
$$

If one or more constraints are satisfied, (21) cannot be computed because $\Delta K$ is singular. In this case, (18) can be minimized sequentially for each nonzero component of $\Delta K=\operatorname{diag}\left(\Delta k_{1}, \ldots, \Delta K\right)$. The corresponding expressions in place of $(20)$ and $(21)$ are

$$
\begin{aligned}
& H^{(0)} \doteq \tilde{H}\left(\sum_{i \in J} Q_{j}\right), J=\left\{i \mid 1 \leqslant i \leqslant p, \Delta k_{j} \neq 0\right\} \\
& Q_{j}=I-\left(\frac{1}{1 / \Delta k_{i}+\left\|g_{i x}\right\|_{\tilde{H}}^{2}}\right) g_{i x}^{*} g_{i x} \tilde{H} .
\end{aligned}
$$

The above results in (20) to (23) were first obtained by kelley, et. a1. [19] by use of a Schur matrix identity. The derivation of this section, however, provides insight into the update's least squares structure and use with an augmented Lagrangian.

Variable metric implementation of the projection-restoration algorithm requires storage of an nxn symmetric matrix. For problems of large dimension, a less costlier 
alternative is offered by another class of conjugate direction methods known as conjugate gradient descent. Implementation is identical to that given in the last section except that the gradient $F_{X}$ is updated before computing the projection increment [3].

\section{NUMERICAL RESULTS}

A computer program based on the above method has been written and applied to various problems. The results reported in this section were obtained on a Telefunken TR 440 computer using double precision arithmetic. Convergence of the algorithm was specified by the stopping conditions

$$
\left\|F_{x}(x, \lambda, K)\right\|_{n}<\varepsilon_{1}, \quad\|g(x)\|_{p}<\varepsilon_{2} .
$$

Resetting of the variable metric matrix $H$ was enforced after every $2(n-p+1)$ projection updates. The "complementary DFP" rank two variable metric update was employed [20] together with a gradient linear search by cubic fit for both the restoration and projection increments. The search required, on the average, four to five function evaluations, i.e. evaluation of $F(x, \lambda, k)$ for specified $x, \lambda$ and $K$. A more accurate linear search had been used in earlier versions of the program without providing significant improvement.

Inequality constraints

$$
g_{j}(x) \leqslant 0, \quad i=p+1, \ldots, p+q
$$

have been accommodated by replacing those elements of $g$ in the penalty terms of (1) by max $\left\{0, g_{j}(x)\right\}$ and by forming the gradients from the $\varepsilon$-active constraints defined by the index set

$$
\left\{i \mid g_{i}(x)+\varepsilon_{j} \geqslant 0, \quad i=p+1, \ldots, p+q\right\}
$$

where $\varepsilon_{i}$ is a small positive constant. This device helps to avoid "jamming" and aids convergence of the algorithm. Further justification is given in the literature [21]. The remaining Kuhn-Tucker condition, that the Lagrange multiplier be non-negative, was not enforced during the descent. In addition, the search was terminated whenever a new constraint is violated.

In the following examples, one cycle is the completion of both a restoration and a projection update. Table 1 lists results for the test problems described below where $N_{C}$ is the total number of cycles required for convergence within $\varepsilon_{1}$ and $\varepsilon_{2} . N_{r}$ and $N_{p}$ are the total number of function evaluations required for building the restoration and projection increments, respectively. The penalty matrix was set to the identity for the initial cycle and updated once. Following the penalty matrix update, the variable metric matrix was updated using (22) and (23). Convergence tolerences are $\varepsilon_{1}=10^{-6}$ and $\varepsilon_{2}=10^{-6}$ for Examples 1 to 4 and $\varepsilon_{1}=10^{-3}$ and $\varepsilon_{2}=10^{-6}$ for Examples 5
and 6 .

Example $1[10]$. The problem is to minimize the functional

$$
f(x)=\left(x_{1}-x_{2}\right)^{2}+\left(x_{2}+x_{3}-2\right)^{2}+\left(x_{4}-1\right)^{2}+\left(x_{5}-1\right)^{2}
$$

subject to linear equality constraints

$$
\begin{aligned}
& g_{1}(x)=x_{1}+3 x_{2}=0 \\
& g_{2}(x)=x_{3}+x_{4}-2 x_{5}=0 \\
& g_{3}(x)=x_{2}-x_{5}=0 .
\end{aligned}
$$

The constrained minimum of $f(\hat{x})=4.0930$ where

$$
\hat{x}=(-.7674, .2558, .6279,-.1163, .2558)
$$

was obtained from a starting point

$$
x=(2,2,2,2,2) .
$$

Example 2 [10]. The problem is to minimize the functional

$$
f(x)=\left(x_{1}-1\right)^{2}+\left(x_{1}-x_{2}\right)^{2}+\left(x_{3}-1\right)^{2}+\left(x_{4}-1\right)^{4}+\left(x_{5}-1\right)^{6}
$$


subject to nonlinear equality constraints

$$
\begin{aligned}
& g_{1}(x)=x_{4} x_{1}^{2}+\sin \left(x_{4}-x_{5}\right)-2 \sqrt{2}=0 \\
& g_{2}(x)=x_{2}+x_{3}^{4} x_{4}^{2}-8-\sqrt{2}=0 .
\end{aligned}
$$

A constrained minimum of $f(\hat{x})=.2415$ where

$$
\hat{x}=(1.1661,1.1821,1.3802,1.5060, .6109)
$$

was obtained from a starting point

$$
x=\{2,2,2,2,2) \text {. }
$$

Example $3[6]$. The problem is to minimize the functional

$$
f(x)=x_{1} x_{2} x_{3} x_{4} x_{5}
$$

subject to the nonlinear equality constraints

$$
\begin{array}{ll}
g_{1}(x)=x_{1}^{2}+x_{2}^{2}+x_{3}^{2}+x_{4}^{2}+x_{5}^{2}-10=0 \\
g_{2}(x)=x_{2} x_{3}-5 x_{4} x_{5} & =0 \\
g_{3}(x)=x_{1}^{3}+x_{2}^{3}+1 & =0 .
\end{array}
$$

A constrained minimum of $f(\hat{x})=-2.9197$ where

$$
\hat{x}=(-1.7171,1.5957,1.8272, .7636, .7636)
$$

was obtained from a starting point

$$
x=(2,2,2,2,2) .
$$

Example 4. The problem is to minimize the functional

$$
f(x)=x_{1}^{2}+9 x_{2}^{2}+9 x_{3}^{2}
$$

subject to the mixed constraints

$$
\begin{aligned}
& g_{1}(x)=x_{1}^{2}+x_{2}^{2}-1=0 \\
& g_{2}(x)=x_{2}+0.5 \leqslant 0 .
\end{aligned}
$$

The constrained minimum of $f(\hat{x})=1$ where

$$
\hat{x}=(0,-1,0)
$$

was obtained from a starting point

$$
x=(2,2,2) \text {. }
$$

Example 5. A nontrivial test of the algorithm is provided by a problem involving the optimal positioning of a geostationary satellite $[22,15]$. We seek a minimum fuel control function and corresponding optimal trajectory subject to dynamic constraints of two body motion, initial state (position and velocity) on a specified transfer orbit, and rendezvous with a specified state on a target orbit. The problem is formulated in a finite dimensional space by requiring control in terms of $N$ impuisive velocity increments which act as equivalent initial conditions for the equations of two body motion. A closed form solution of the resulting initial condition problem and the forward sensitivity matrices due to Goodyear enables accurate evaluation of the constraints and their gradients over the trajectory. The parameter set is the $4 \mathrm{~N}$-tuple

$$
\pi=\left(t_{1}, \ldots, t_{N}, c_{1}, \ldots, c_{N}\right)
$$

consisting of the switching times and velocity increments, respectively. Six equality constraints are obtained from the rendezvous requirement. Additional inequality constraints specify bounds on the switching times and magnitudes of the velocity increments.

In this example, the algorithm is used to compute the positioning control using three impulses $(n=12)$ for the following input data. Transfer orbit: semi major axis, $a=25078 \mathrm{~km}$, eccentricity, $e=.736047$; inclination, $i=5^{\circ}$; argument of perigee, $\omega=180^{\circ}$; longitude of the ascending node, $\Omega=180^{\circ}$; eccentric anomaly, $E\left(t_{A}\right)=180^{\circ}$. Target orbit: position, $r\left(t_{A}\right)=(-42164.22,0,0) \mathrm{km}$ and velocity, $v\left(t_{A}\right)=(0,-3074.65,0) \mathrm{m} / \mathrm{sec}$ of the 
rendezvous point where $t_{A}$ is the time of apogee passage in the transfer orbit. Position is relative to an earth centered coordinate system with the $x-y$ axis in the plane of the equator. In addition to the six rendezvous (equality) constraints, an inequality constraint was specified by the requirement that the first velocity increment not exceed $1440 \mathrm{~m} / \mathrm{sec}$.

Using a starting point of

$$
\begin{array}{ll}
t_{1}=0 \mathrm{hr} & c_{1}=(0,-1420,0) \mathrm{m} / \mathrm{sec} \\
t_{2}=12 & c_{2}=(0,-50,0) \\
t_{3}=24 & c_{3}=(0,-20,0),
\end{array}
$$

the optimal control parameters obtained were

$$
\begin{array}{ll}
\hat{t}_{1}=.017 & \hat{c}_{1}=(1.94,-1435.6,-111.9) \\
\hat{t}_{2}=11.33 & \hat{c}_{2}=(17.6,-29.3,16.7) \\
\hat{t}_{3}=22.8 & \hat{c}_{3}=(-1.06,-14.5,-8.5),
\end{array}
$$

corresponding to a minimum fuel cost of $1494.9 \mathrm{~m} / \mathrm{sec}$. A more detailed description of this and related examples is given in reference [15].

Example 6. A second application of the algorithm to trajectory optimization is provided by a proposed rendezvous with a comet [23]. For scientific investigation of the comet as it approaches the sun, it is required that the maximum allowable fly-by velocity of the spacecraft be sma17. By defining the cost functional as the velocity increment required for injection from Earth orbit plus that required for rendezvous, a solution yielding the minimum fuel for the best fly-by is obtained. The impulsive trajectory formulation used in Example 5 is applied and the algorithm is used to compute the optimal parameters using two impulses $(n=8)$ for the following input data. Initial (Earth) orbit: $a=1.5 \times 10^{8} \mathrm{~km}, e=.0167, i=0^{\circ}, \omega=101.2208^{\circ}, \Omega=0^{\circ}$, and the mean anomaly $M\left(t_{a}\right)=-101.2208$ where $t_{a}=0$ corresponds to an epoch of September $23,1980$. Final orbit: $a=3.3225 \times 10^{8} \mathrm{~km}, e=.8462, i=12.35^{\circ}, \omega=185.2^{\circ}, \Omega=334.72^{\circ}, M\left(t_{b}\right)=0$ where $t_{b}=0$ corresponds to an epoch of December 12, 1980. Six equality constraints are specified by the rendezvous requirement.

Using a starting point of

$$
\begin{array}{ll}
t_{1}=0 \text { days } & c_{1}=(-5.9,-5.9,0) \mathrm{km} / \mathrm{sec} \\
t_{2}=100 & c_{2}=(-11,-13,4),
\end{array}
$$

the optimal control parameters obtained were

$$
\begin{array}{ll}
\hat{t}_{1}=-24.75 & \hat{c}_{1}=(-3.005,-8.421,4.182) \\
\hat{t}_{2}=81.16 & \hat{c}_{2}=(-1.102,-6.539,-2.416),
\end{array}
$$

corresponding to a launch date of August 29, 1980 and a flight time of 106 days. The injection velocity is $9.87 \mathrm{~km} / \mathrm{sec}$ and the fly-by velocity is $7.05 \mathrm{~km} / \mathrm{sec}$ (Figure 1 ).

\section{CONCLUSIONS AND COMMENTS}

Although the algorithm is heuristic in the sense that convergence criteria are not provided, numerical experiments by the authors indicate that the program is generally more reliable than that employing only gradient projection or penalization alone. The main difficulty to date, and one that is familiar to users of penalty function techniques, is the proper choice and updating of the penalty constants. However, bounds on the magnitudes of the constants are available [11].

The algorithm has also been applied to a problem involving the optimal control of heat distribution at the interface of an inhomogeneous rectangular solid. In view of the large number of parameters resulting from time and spatial discretization of the control function, the gradient of the cost is most efficientiy evaluated from the state and adjoint (backward sensitivity) equations. In this case, the Green's function for the system and adjoint are simply related due to the assumed linearity of the model and can be explicitly obtained in terms of a Fourier series. 


\section{ACKNOWLEDGEMENT}

The work of W. A. Gruver was supported through a Senior Scientist Award administered by the U. S. Special Program of the Alexander von Humboldt Foundation.

\section{REFERENCES}

1. Lootsma, F. A.: A Survey of Methods for Solving Constrained Minimization Problems via Unconstrained Minimization, in F. Lootsma (ed), Numerical Methods for Nonl inear Optimization, Academic Press, New York, 313-347 (1972).

2. Rosen, J. B.: The Gradient Projection Method for Nonl inear Programming; Part I: Linear Constraints, J. SIAM, 8, 181-217 (1960); Part II: Nonlinear Constraints, J. SIAM, $\underline{9}, 414-443$ (1961).

3. Miele, A., Huang, H., and Heideman, J.: Sequential Gradient-Restoration Algorithm for the Minimization of Constrained Functions - Ordinary and Conjugate Gradient Versions, J. Optimization Theory and Applications, 4, No 4, 213-242 (1969).

4. Kelley, H. J. and Speyer, J. L.: Accelerated Gradient Projection, in Lectures in Mathematics, 132, Springer Verlag, Ber1 in-Heidelberg, 151-158 (1970).

5. Hestenes, M. R.: Multiplier and Gradient Methods, in L. Zadeh (ed), Computing Methods in 0ptimization Problems, 2, Academic Press, New York, 143-163 (1969).

6. Powe11, M. J. D.: A Method for Nonlinear Constraints in Minimization Problems, in R. Fletcher (ed), Optimization, Academic Press, New York, 283-298 (1969).

7. Roode, J. D.: Generalized Lagrangian Functions in Mathematical Programming, Thesis, University of Leiden, Netherlands, (1968).

8. Rockafellar, R. T.: Augmented Lagrange Multiplier Functions and Duality in Nonconvex Programming, SIAM J. of Control, to appear.

9. Haarhoff, P. C. and Buys, J. D.: A New Method for the Optimization of a Nonlinear Function Subject to Nonlinear Constraints, Computer J., 13, 178-184 (1970).

10. Miele, A., Cragg, E., Iyer, R., and Levy, A.: Use of the Augmented Penalty Function in Mathematical Programming Problems, Part 1, J. Optimization Theory and Applications, 8, 115-130 (1971).

11. Martensson, K.: Methods for Constrained Function Minimization, Report 7107, Div. of Automatic Control, Lund Institute of Technology, Sweden, March 1971.

12. Glad, T.: Lagrange Multiplier Methods for Minimization Under Equality Constraints, Report 7323, Div. of Automatic Contro1, Lund Institute of Technology, Sweden, August 1973.

13. Tripathi, S. S. and Narendra, K. S.: Constrained Optimization Problems Using Multiplier Methods, J. Optimization Theory and Applications, 9, 59-70 (1972).

14. Wierzbicki, A. P.: A Penalty Function Shifting Method in Constrained Static Optimization and $i$ ts Convergence Properties. Archiwum Automatyki $i$ Telemechaniki, 16, 395-416 (1971).

15. Gruver, W. A. and Engersbach, N. H.: Nonl inear Programming by Projection-Restoration Applied to Optimal Geostationary Satellite Positioning, AIAA Journal, December 1974. 
16. Engersbach, N. H. and Gruver, W. A.: Constrained Optimization Based on Generalized Exterior Point Methods, Report IB013-72/10, Deutsche Forschungs- und Versuchsanstalt für Luft- und Raumfahrt, December 1972.

17. Broyden, C. G.: Quasi-Newton Methods, in W. Murray (ed), Numerical Methods for Unconstrained Optimization, Academic Press, 87-106 (1972).

18. Goldfarb, D.: Extension of Davidon's Variable Metric Method to Maximization Under Linear Inequality and Equality Constraints, SIAM J. Applied Math., 17, 739-764, July 1969.

19. Kelley, H. J., Denham, W., Johnson, I., and Wheatley, P.: An Accelerated Gradient Method for Parameter Optimization with Nonl inear Constraints, J. Astronautical Sciences, 13, No 4, 166-169, Ju7y-August 1966 .

20. Goldfarb, D.: A Family of Variable-Metric Methods Derived by Variational Means, Maths. Computation, 24, 23-26 (1970).

21. Zangwill, W. I.: Nonlinear Programming, Prentice-Hal1, Englewood Cliffs, N. U., Chapter 13 (1969).

22. Gruver, W. A. and Engersbach, N.: A Mathematical Programming Approach to the Optimization of Constrained, Impulsive, Minimum-Fuel Trajectories, Report IB01372/3, Deutsche Forschungs- und Versuchsanstalt für Luft- und Raumfahrt, June 1972.

23. Eckstein, M. C. and Jochim, E. F.: Vorläufige Untersuchung zur Bahnoptimierung für die Helio-C Mission, Report IB522-73/1, Deutsche Forschungs- und Versuchsanstalt für Luft- und Raumfahrt, March 1973.

TABLE 1

CONVERGENCE PROPERTIES OF THE PROJECTION-RESTORATION METHOD

\begin{tabular}{|c|c|c|c|c|c|c|c|}
\hline \multirow[t]{2}{*}{ Problem } & \multirow{2}{*}{$\begin{array}{c}\text { Cycles } \\
\mathrm{N}_{\mathrm{C}}\end{array}$} & \multirow{2}{*}{$\begin{array}{l}\text { Function } \\
\qquad N_{r}\end{array}$} & \multirow{2}{*}{$\begin{array}{l}\text { Evaluations } \\
\qquad N_{p}\end{array}$} & \multirow{2}{*}{$\begin{array}{l}\text { CPU Time } \\
\text { sec }\end{array}$} & \multirow{2}{*}{$\begin{array}{c}\text { Parameters } \\
n\end{array}$} & \multicolumn{2}{|c|}{ Constraints } \\
\hline & & & & & & $\mathrm{p}$ & $q$ \\
\hline 1 & 4 & 20 & 20 & 0.668 & 5 & 3 & \\
\hline 2 & 11 & 39 & 66 & 1.784 & 5 & 2 & \\
\hline 3 & 5 & 27 & 25 & 0.902 & 5 & 3 & \\
\hline 4 & 7 & 36 & 39 & 1.350 & 3 & 1 & 1 \\
\hline 5 & 16 & 61 & 75 & $33^{b}$ & 12 & 6 & 1 \\
\hline 6 & 15 & 51 & 75 & $20^{b}$ & 8 & 6 & \\
\hline
\end{tabular}

a Telefunken TR 440 Computer (double precision).

Includes evaluation of dynamical system constraints and gradients. 


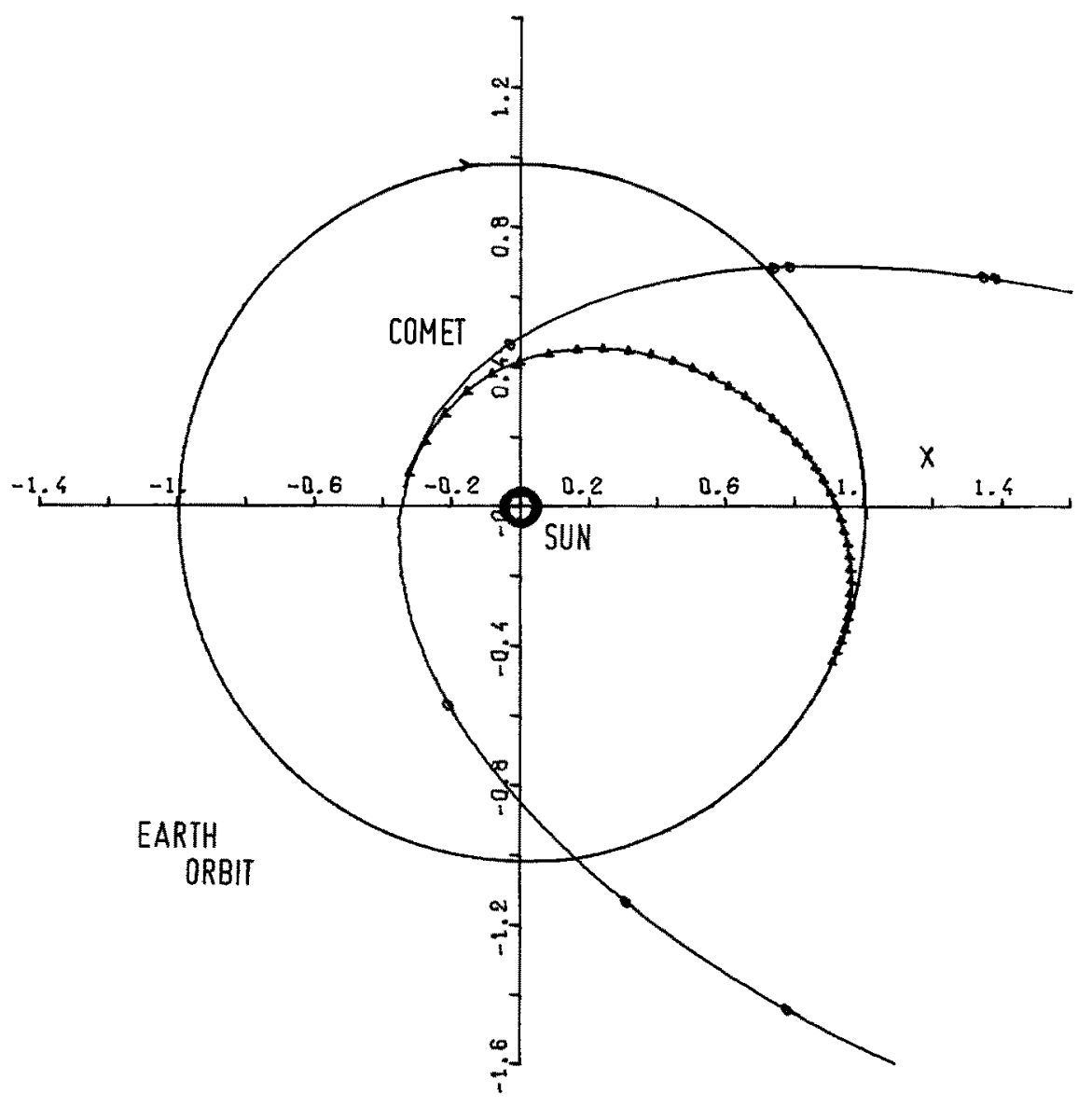

FIGURE 1. RENDEZVOUS COMET ENCKE 1980 